\title{
Einstein's Misunderstanding of Time in the Time-Invariant Universe
}

\author{
Amrit S. Šorli * \\ Bijective Physics Institute, Slovenia \\ sorli.bijective.physics@gmail.com
}

\section{Štefan Čelan}

Scientific research centre Bistra, Slovenija

stefan.celan@bistra.si

Received Jan 2021

Received in revised: Jan 2021

Published: March 2021

\begin{abstract}
ABSTRA CT
Einstein has kept time as the dimension of the space-time continuum that is supposed to be a fundamental arena of the universe. Our research confirms time is the duration of changes, i.e., motion run in the time-invariant universal space that has Euclidean shape, it is infinite. Black holes in the centre of galaxies are rejuvenating systems of the universe. In these black holes old matter is transforming back into the fresh energy of elementary that AGNs are throwing in the intergalactic space in the form of huge jests. These jets are fresh material for new star formation. The universal process of continuous rejuvenation is eternal.
\end{abstract}

Keywords: Space, Time, Cosmology, Olbers’ paradox, AGN-s.

C2021 The Authors. Published by Fundamental Journals. This is an open access article under the CC BY-NC

https://creativecommons.org/licenses/by-nc/4.0/

\section{INTRODUCTION}

The model of space-time as the fundamental arena of the universe is being replaced by the model of the time-invariant universal space, where time is merely the duration of change, i.e., motion (Šorli \& Čelan, 2020).

In time-invariant universal space time is not its $4^{\text {th }}$ dimension. Experimental physics confirms this view, with clocks we measure the duration of a material change, i.e., motion in space. Moreover, the rigorous analysis of Special Relativity formalism of the fourth coordinate of space-time confirms that the fourth coordinate $X_{4}$ is not time $t$

$$
X_{4}=i c t
$$

In Eq. (1) $i$ is the imaginary unit, $c$ is the light speed, and $t$ is duration.

In Eq. (2) the time $t$ is the duration of photon motion in space, time $t$ is not $X_{4}$ (Fiscaletti \& Sorli, 2017) 


$$
t \neq X_{4}
$$

Einstein has interpreted the time $t$ as the $4^{\text {th }}$ coordinate $X_{4}$ of a Minkowski manifold.

He wrote: "If we replace $x, y, z, \sqrt{-1} c t$ by $x_{1}, x_{2}, x_{3}, x_{4}$, we also obtain the result that $d s^{2}=d x_{1}^{2}+d x_{2}^{2}+d x_{3}^{2}+d x_{4}^{2}$ is independent of the choice of the body of reference. We call the magnitude $d s$ the "distance" apart of two events or fourdimensional points. Thus, if we choose as time variable the imaginary variable $\sqrt{-1} c t$ instead of the real quantity $t$, we can regard the continuum space-time, in accordance with the special theory of relativity, as an "Euclidean" fourdimensional continuum, a result following by the consideration of the preceding section" (Einstein, 1920).

In the above citation, Einstein suggestions that we can choose the time variable $t$ as the imaginary variable, can be written as follows:

$$
t=\sqrt{-1} c t
$$

Eq. (3) is false because on the left side of the equation we have $t$ and on the left side we have $\sqrt{-1} c t$. Combining Eq. (3) with equation well known equation $X_{4}=i c t$ we get,

$$
X_{4}=i t c^{2} \sqrt{-1}
$$

Equation (4) confirms Einstein did a mistake keeping and interpreting time as the dimension of a four-dimensional continuum. Physics is still today suffering this misinterpretation of time that is solved in this article: time is the duration of material change, i.e., motion in time-invariant space.

Considering time as the duration of a change running in space, the universal space results as being time-invariant; the duration of a given event running in the universal space does not change in any way the physical properties of space and is not a part of the space. NASA has measured in 2014 that the universe space has Euclidean shape, measure the angles between three stellar objects and getting $180^{\circ}$ with $0.4 \%$ margin of error. This means that the universe has Euclidean shape and is infinite (NASA, 2014).

The idea of time-invariant structure of the universe was recently presented also by Hans J. Farr and Michael Hey: "One more cosmological possibility might perhaps need to be considered here, namely that the hierarchical structuring of masses in the universe which was considered in the above calculation could perhaps also be a time-invariant cosmic structuring, meaning that even though the universe undergoes an expansion in cosmic time, its hierarchical structuring endures or persists. Of course, an expanding hierarchical universe must also change its mass density, however in such a way that the hierarchical structuring of matter persists, i.e., a time-invariant scale-invariance under these auspices must be considered" (Fahr \& Heyl, 2020).

The right understanding of time as the duration of change, i.e., motion in time-invariant space is in our view one of the most important elements of 21 st-century physics progress.

\section{UNIVERSE IS TIME-INVARIANT AND ETERNAL}

Today's cosmology examines the universe from the perspective of the universe is existing in some linear time that has physical existence. We are seeing the universe as something that has started long ago and is still developing in the present day. A rigorous examination of what is time confirms that time as the duration enters existence only when measured by the observer. There is no physical time running in the physical universe on its own. Universal changes are irreversible.

When change $X+1$ enters existence, change $\mathrm{X}$ is not in existence anymore. When change $X+2$ enters existence, change $X+1$ is not in existence anymore. Changes run in a time-invariant universal space where there is no past, there is no present and there is no future. The linear time "past-presentfuture" exists only in the human brain, it has its physical origin in neuronal activity (Šorli \& Čelan, 2020)

The only universe that exists is the one we can observe and measure. NASA has measured universal space has a Euclidean shape and is infinite. We are living in an infinite time-invariant universe where there is no physical past and there is no physical future.

From this perspective, it makes no sense to build a hypothesis about the begging of the universe in some remote physical past because such a past is non-existent. Would be more opportune to build cosmology only on the basis of astronomical observation and without hypothetical speculations about some remote beginning in some remote physical past; the universe is time-invariant which means eternal.

There was no beginning and there will be no end. Active galactic nucleus (AGN) in the centre of galaxies is transforming old matter into fresh energy in the form of elementary particles. These particles are forming huge jets that are thrown out in the intergalactic space.

Black holes in the centre of galaxies are rejuvenating systems of the universe that is eternal without the beginning and without an end (Šorli \& Čelan, 2021).

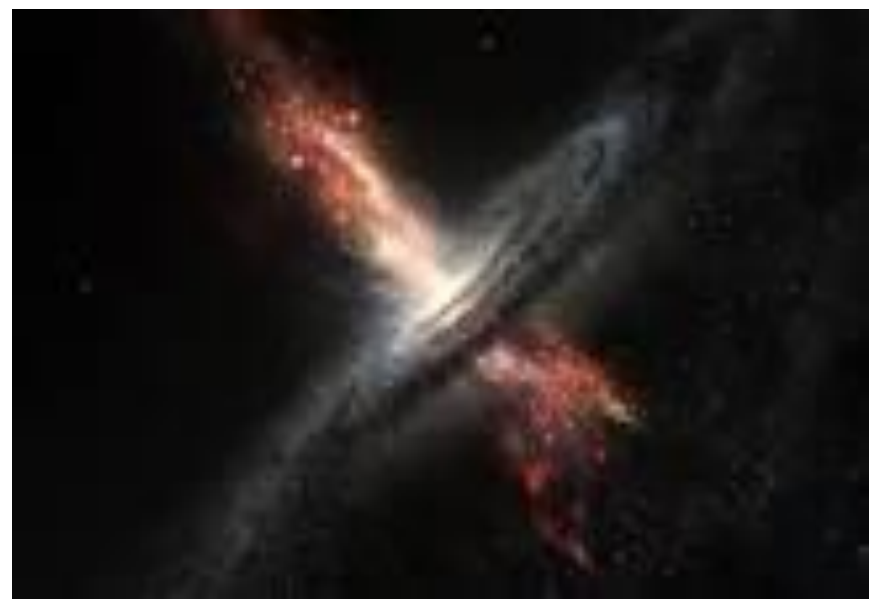

Figure 1: Jets from the black hole in the centre of galaxy (with permission of European Southern Observatory - ESO) 
The universe is eternal and this eternity is NOW. Humans, we experience the time-invariant nature of the universal space as NOW. Eternity is not extending infinitely back into the past and is not extending infinitely ahead in the future. Past, present and future exist only in the human mind; eternity is NOW (Fiscaletti \& Sorli, 2014).

Intuitively Einstein knew this and he expressed this in the following famous words: "People like us who believe in physics know that the distinction between past, present, and future is only a stubbornly persistent illusion". Still, in physics, he kept time as the fourth dimension of the model of the spacetime continuum. The space-time continuum model has no correspondence in the physical world where we observe only material changes running in time-invariant space. Time as duration enters the existence when we me measure it.

\section{Olbers' PARADOX SOLUTION}

The formalism for the luminosity of a star at a finite distance from the Earth is as follows.

$$
L=b 4 \pi d^{2}
$$

where $b$ is the apparent brightness of the star, $L$ is its luminosity, and $d$ the distance to the star. As a solution for Olbers' paradox, I argue in this way: the luminosity of stars which are in the area of finite distance from us is too low to make a day when we have a night. The light from the stars which are at infinite distance will never reach us and so cannot make a day when we have a night.

Harisson definition of the Olbers' paradox is as follows: "Olbers in 1826 was the first to show that the radiation density everywhere in an infinite static universe should equal to the radiation density at the surface of the stars. Hence, Olbers' paradox is that the sky is dark at night" (Harrison, 1964).

Olbers did not take into account that only the light from stars that are at a finite distance from the Earth can reach us.

The light of stars that are infinitely far away cannot reach us, and that of stars at a finite distance is not strong enough to make the night a day.

This is also the conclusion of Harrison article back in 1964: "Thus the radiation-level is low in our universe at present and the night-sky is dark simply because the stars are so widely separated from each other, or, in other words, because the characteristic time $\tau_{0}$ is so very large (Harrison, 1964).

Our comment on the last Harrison's citation is that time in the solution of Olbers' paradox does not count. Time is the duration of light motion from the stars to the Earth. Time here does not play any role in Olbers' paradox solution. The decisive role is played by the distance and the luminosity of stars; the luminosity of stars at a finite distance is not strong enough to make a night sky a day.

The light from stars that are at an infinite distance will never reach us and does not count.

Sakar's and Jeffries's research suggests that the paradox is resolved by the fact that the universe is expanding, which means that distant light has not yet reached us (Sarkar \& Jeffries, 2002).
Taking into account that the universe is expanding, our solution is still valid, namely, that the light of stars which are infinitely way from us will never reach us and the light of stars on a finite distance is not strong enough to make of a night sky a day. Knutsen's research suggests: "It is dark at night because:

(1) The speed of light is finite;

(2) The universe is still young, and the stars only started to shine rather recently;

(3) The light sources in the universe are small;

(4) The light sources in the universe are far apart, so the energy density in the universe is very small.

Both obscuration and expansion are quite unimportant" (Knutsen, 1997). None of these conclusions above is an exact explanation. It is dark at night because the light coming from the stars at a finite distance is not strong enough to make a night sky a day.

Harari's research suggests: "For terrestrial observation, when we consider the resolution of Olbers' paradox, we can use the apparent magnitude 8 as a conservative limiting magnitude. Thus, we can restate our earlier conclusion by noting that any star that has an apparent magnitude of fainter than 8 will not be visible to the average unaided human eye, and can, therefore, be considered non-existent for the analysis of the cumulative apparent brightness of the night sky under observation by the unaided eye.

This limiting apparent magnitude constraint acts as a high-pass apparent brightness filter that effectively removes all fainter stars from the population of visible stars as far as unaided eye vision is concerned, thereby rendering finite the population of visible stars, irrespective of the initial size of the population of stars that exist in the universe, a population that may indeed be infinite"(Harari, 2019).

Harari's solution of Olber's paradox is tuned with the solution proposed in this article: the stars in the universe that have an apparent magnitude below 8 and are at a finite distance from the Earth are invisible to the unaided human eye, which means that the luminosity of stars at a finite distance is not strong enough, to make the night sky a day.

Olber's solution is deeply related to tour understanding of infinity. Infinite distance plus $1000 / \mathrm{km}$ still is an infinite distance. "infinite distance" is not a metrical term that would describe some distance from our position in space to some physical object far away in the space.

From the mathematical point of view, this is the problem of infinite numbers posed by German mathematician Georg Cantor: is an infinity of real numbers bigger than an infinity of natural numbers? Slovenia mathematician Ivan Vidav solved this problem proving that if we say that the infinity of real numbers is bigger than the infinity of natural numbers, there is no contradiction (Vidav, 1959).

If we say that both infinities are equal there also there is no contradiction. This clearly confirms that infinity is not a metrical term. In this perspective human imagination of the size of the universe is limited. We do not know exactly what infinity means, but one thing is clear: no light from the stars that are in infinity can reach us. 


\section{THE TIME-INVARIANT SPACE MODEL SURPASSES} PRESENTISM AND EXTERNALISM

The result of our research is the replacement of the space-time model with the time-invariant space model as the fundamental arena of the universe. There is no such thing in the universe as space-time and "slices of space-time".

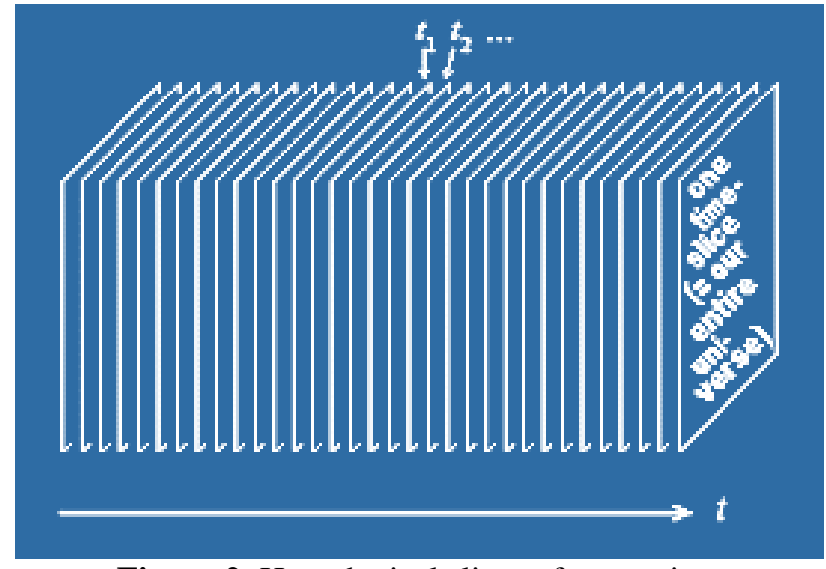

Figure 2: Hypothetical slices of space-time

There is no such thing in the universe as some physical "time distance" $\Delta t$ between two events.

$$
\Delta t=t_{2}-t_{1}
$$

where $t_{1}$ is a given slice of space-time and $t_{2}$ is the next slice of space-time (see Fig 3 ). The duration between two events $\Delta t$ enters into existence in the act of the measurement from the side of the observer. All events in the universe happen in the same identical time-invariant space. Clocks tick in timeinvariant space. Clocks do not measure some physical time running on their own. Duration enters existence when measured by the observer.

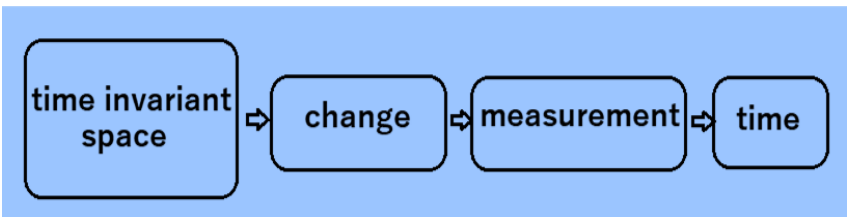

Figure 3: Time is the result of measurement with clocks

The universe is time-invariant. It does not run in some physical time; it runs in time-invariant space. Universal changes are irreversible, physical past is non-existent. Future also is nonexistent. Time travel is out of the question. One can only travel in time-invariant space. Presentism believes that past and future are somehow coexisting in the present moment that is the only moment that exists. The entire history and the entire future of the universe are squished in the present moment. Eternalism believes that time is extending infinitely far into the past and infinitely far into the future. We can imagine presentism as the mathematical point and Eternalism as the infinite straight line. We will examine both views from the pragmatic view. You take a stone, you keep it above your leg, and then you throw it on your leg. When the stone hits you on the leg, you feel the pain. Before you lift the stone from the ground there was no pain in your leg. This proves that in the present moment events are not coexisting. They are following each order in the sequential order: 1. lifting the stone, 2. keeping the stone above the leg, 3. throwing the stone on the leg. We have seen in chapter 2 that sequential events in the universe are irreversible and are not coexisting.

Presentism seems wrong; it is not the truth that everything coexists in the present moment. Eternalism sees the event with the stone is happening in the linear physical time one after other. Eternalism keeps the past as something real despite nobody ever reach into the past. For Eternalism, the universe runs in some linear time that nobody ever measured and observed. Seems this view is not right.

In cosmology, presentism is an inspiration for the "block universe" model where everything that happens is coexisting. Eternalism is the inspiration for the "space-time continuum" where we can have "closed time-lines" discovered by Kurt Gödel. The closed time-lines theoretically allow one could travel back in time and kill his grandfather and so it could not be born. That's why Gödel said: "In any universe described by the Theory of Relativity, time cannot exist" (Sorli, Fiscaletti, \& Gregl, 2013).

Gödel's discovery is still today interpreted wrong by some researchers who think that his development of General Relativity equations and consequently the discovery of closed time-lines indicates that one could travel back in time. On the contrary, Gödel was strictly against the idea of time travel. One can travel only in time-invariant space where there is no present, no past, and no future. In the time-invariant space model, there is no physical time in the sense of present time as considered in presentism and there is no time in the sense of some linear physical time as considered in Eternalism. Time as duration enters existence when measured by the observer. Time-invariant space that we humans experience as the present moment is the fundamental non-created eternal background of the universe. Universal changes run in this time-invariant space that is eternity itself. Humans experience the flow of changes in the frame of psychological time "past-presentfuture" and that's why we experience changes running in some linear physical time that is not there. We are "projecting" our psychological linear time that is the product of neuronal activity in the physical universe (Šorli \& Čelan, 2020).

In the universe, we can only observe the relative rate of clocks and not some "relative time". Clocks run on the GPS satellites for 45 microseconds per day faster than on the Earth surface because of the General Relativity effect. And they run slower for 7 microseconds per day because of the SR effect (Ashby, 2002). Clocks tick only in time-invariant space and not in some physical time. What is "relative" in the universe is not time, relative is the rate of clocks and velocity of material changes in general. A twin on the Moon would age faster than his brother on the Earth's surface because the velocity of changes on the Moon is a bit faster regarding the Earth's surface. The weaker is gravity faster is the rate of clocks and aging too. In interstellar space where gravity is weak, the twin would age a bit faster than his brother on the Moon surface or on Earth surface. But there is no "twin paradox". Twins are aging in time-invariant space. 


\section{CONCLUSIONS}

In today's physics we still think that with rope we measure distance in space and with clocks we measure distance in time. Einstein has kept time as the dimension of a four-dimensional continuum.

\section{REFERENCES}

Ashby, N. (2002). Relativity and the global positioning system. Physics Today, 55(5), 41-47. https://doi.org/10.1063/1.1485583

Einstein, A. (1920). Relativity: The Special and General Theory (Methuen \& Co., London, 1920), Chap. XXII. 11The International System of Units (SI), edited by BN Taylor, NIST Special Publication(330), 5.

Fahr, H. J., \& Heyl, M. (2020). A universe with a constant expansion rate. Physics \& Astronomy International Journal, 4(4), 156-163. 10.15406/paij.2020.04.00215

Fiscaletti, D., \& Sorli, A. (2017). Searching for an adequate relation between time and entanglement. Quantum Studies: Mathematics and Foundations, 4(4), 357-374. doi: 10.1007/s40509-017-0110-5

Fiscaletti, D., \& Sorli, A. S. (2014). The Infinite History of NOW: A Timeless Background for Contemporary Physics: Nova Publishers.

Harari, Z. (2019). Analytical Resolution of the Dark Night Sky (Olbers') Paradox. Astronomische Nachrichten, 340(6), 510-524. https://doi.org/10.1002/asna.201913540

Harrison, E. (1964). Olbers' paradox. Nature, 204(4955), 271-272. https://doi.org/10.1038/204271b0
Our research confirms that this space-time continuum does not exist in the physical reality. Irreversible universal changes run in time-invariant universal space where black holes in the centre of galaxies are rejuvenating systems of the universe that is eternal and non-created.
Knutsen, H. (1997). Darkness at night. European Journal of Physics, 18(4), 295. https://doi.org/10.1088/01430807/18/4/010

NASA. (2014). from https://wmap.gsfc.nasa.gov/universe/uni_shape.html

Sarkar, S., \& Jeffries, B. (2002). The solution to Olbers' paradox. Physics World, 15(10), 17. https://doi.org/10.1088/2058-7058/15/10/27

Sorli, A., Fiscaletti, D., \& Gregl, T. (2013). New insights into Gödel's universe without time. Physics Essays, 26(1). https://doi.org/10.4006/0836-1398-26.1.113

Šorli, A. S., \& Čelan, Š. (2020). The End of Space-time: Physics-Mathematics. International Journal of Fundamental Physical Science, 10(4), 31-34. https://doi.org/10.14331/ijfps.2020.330139

Šorli, A. S., \& Čelan, Š. (2021). Schwarzschild energy density of superfluid quantum space and mechanism of AGNs' jets. Advanced Studies in Theoretical Physics, 15(1), 9-17.

Vidav, I. (1959). Rešeni in nerešeni problemi matematike: Mladinska knjiga. 